\title{
Characterizing User Groups in Online Social Networks
}

\author{
László Gyarmati and Tuan Anh Trinh \\ High Speed Networks Lab \\ Department of Telecommunication and Media Informatics \\ Budapest University of Technology and Economics \\ \{gyarmati, trinh\}@tmit.bme.hu
}

\begin{abstract}
The users' role is crucial in the development, deployment and the success of online social networks (OSNs). Despite this fact, little is known and even less has been published about user activities in the operating OSNs. In this paper, we present a large scale measurement analysis of user behaviour, in terms of time spent online, in some popular OSNs, namely Bebo, Flixster, MySpace, and Skyrock, and characterise user groups in OSNs. We used more than 200 PlanetLab 1 nodes for our measurement, monitored more than 3000 users for three weeks by downloading repeatedly their profile pages; more than 100 million pages were processed in total. The main findings of the paper are the following. Firstly, we create a measurement framework in order to observe user activity. Secondly, we present cumulative usage statistics of the different OSNs. Thirdly, we classify the monitored users into different groups and characterise the common properties of the members. Finally, we illustrate the wide applicability of our datasets by predicting the sign out method of the OSN users.
\end{abstract}

\section{Introduction}

Online social networks change the way how humans connect, get in touch with each other. Novel online social networks are published almost every day but only a few of them become popular worldwide, the most of them vanish. The success of a social network - both short-term and long-term - depends on the behaviour of its users, in particular the users' activity has an important effect on the services.

The activity of the users, time spent on the OSN, is an important aspect if the value of the social networks has to be expressed. Only the operators of the OSNs have these informations although investors, seeding firms, and advertisers would appreciate and utilize this knowledge. In addition, developers of new social networks can build their systems with more incentives if they merge the properties of successful OSNs.

Measurement studies of online social networks are starting to build up in the last few years. The first group of the results deals with the topology and structure of OSNs. For example, the topological properties of several online social 
networks are examined based on real world measurements in [2], including verifying the scale-free network property. Similarly, the topology of Flickr and and its growth is covered in 3 . The second group deals different aspects of contents and applications in OSNs. The authors of 45] characterise the usage of social network based applications. User behaviour in user generated content video systems are analysed in [6, based on the popularity of the videos. Furthermore, the structure of blog entries interaction and social connections are explored in [7], while [8] investigated information spreading in OSNs.

These studies improve understanding the characteristics of OSNs, both structurally and functionally, however the measurement of user activity, e.g. time spent online by the users, on OSNs has received little attention from research community so far. In this paper, we present a large scale measurement analysis of user behaviour in some popular OSNs. We used more than 200 PlanetLab nodes for our measurement, monitored more than 3000 users for three weeks by downloading more than 100 million profile pages. We traced members of multiple OSNs, in particular users of MySpace [9], Bebo [10, SkyRock [11, and Flixster [12, to identify common properties of online social networks and identify user groups in OSNs. We obtained our data by processing publicly accessible user profiles with a minute as sampling time over more than three weeks, we make our measurement data available to the research community.

The paper is structured as follows. We describe the goals and challenges of our measurement and the methodology for downloading and processing the social networks in Section 2. Section 3 present an in-depth statistical analysis of user activity on OSNs. In particular, after revealing high-level statistics we classify the users of OSNs into several groups. After identifying the properties of the clusters, we present a method to predict the way a user sign off from the OSN, as an illustration the wide-range applicability of our datasets. Finally, we conclude in Section 4 .

\section{Measurement Methodology}

This section describes the goals of our measurement by identifying the main challenge of an Internet-scale measurement. In addition, we present what type of data have been obtained, how have we collected and processed them.

\subsection{Measurement Goals and Challenges}

In this section, we describe the non-technology related aspects of our measurement. Our research focus is the user activity on online social networks, therefore datasets that contain individual, not aggregated, online status information of OSNs' users are required. We were not able to obtain data directly from the operators of the OSNs, they only referred to the measurements of comScore [13. which contains only an average, monthly usage data, neither individual nor dynamic user activity metrics are presented. Therefore, we iteratively downloaded the public part of users' profile pages, which contain online status information, 
and collected real world usage datasets to discover common usage patterns and to highlight the differences of OSNs, instead of carrying out a survey by asking users of OSNs about their usage habits.

The online status of users is handled differently by OSNs: some OSNs do not display status information, some OSNs display it for authorised members (e.g. friends, group members, or logged-in users) while the others handle it publicly if this is allowed by the user. We selected four OSNs where the status information is publicly available. In order to create a statistical analysis of the user activity, the size of the sample (the monitored users) has to be selected appropriately, furthermore the sampling time of the measurement has to be selected carefully because of feasibility problems as the time of downloading and processing a single user is not negligible. Accordingly, we decided to monitor around 1000 users per OSNs on a one minute basis as a trade-off of accuracy and feasibility. The exact number of the monitored users were determined by the availability of the computers used in the measurement. If a user spends less than a minute online it might not be identified as an online user, however, we believe this is only a minor limitation of our measurement.

We tried to select users to be monitored to catch the characteristic of newly joined members of the OSNs, who spend their time on site in order to make connections. However, only MySpace and SkyRock allow to list their new members, on the other sites randomly selected users were monitored.

As several additional properties may have an effect on the activity of the users, we extracted not only the uptime, the time spent online, of the users but - if it is presented - the number of friends and their membership history. Other, nonmeasurable factors may have an impact on users activity, including uncertainties of human decisions, weather, or professional activities, which we do not and can not incorporate to our measurements.

Our trace do not contain information about the correlation of activities of connected members, or members of user groups, these topics should be investigated in the future. In addition, the users of OSNs may use more than one sites to connect with their friends; an ineresting future work can analyse and reveal the common parts of personal behaviour across multiple OSNs. The datasets of our measurement are available at http://netecon_group.tmit.bme.hu/source-codes [14] where the user identifiers are replaced for anonymisation purposes.

\subsection{Measurement Setup and Tool}

We now discuss the technical aspects of our measurement, including profile page downloading and processing, and we present our monitoring tool. We monitored more than 3000 users, selected randomly, of four OSNs during our measurement on a minute basis, while downloading and processing a typical profile page is around a second. The measurement was carried out between 28 January and 20 February, 2009. Based on the theorem of statistics, the size of the monitored users is large enough to draw conclusion, e.g. political polls are also usually use samples of around 1000 persons. However, we note that our measurement sample 


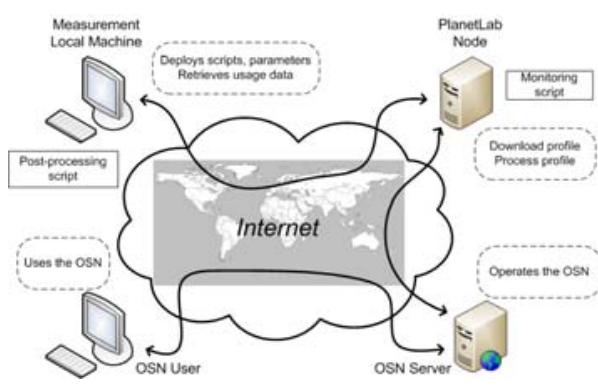

(a) Measurement setup

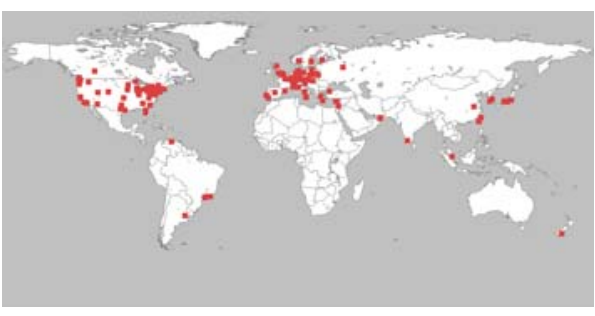

(b) Topology of PlanetLab nodes used

Fig. 1. Overview of the measurement

might not be a representative sample as OSNs do not publish information about the structure of their population publicly.

Accordingly, the monitoring of users can not be carried out from a single, standalone machine because of scalability reasons. Therefore, we used 212 nodes of PlanetLab, a cluster of more than 900 machines, to carry out the measurement. The machines of PlanetLab executed automated, timed scripts, implemented in Python, which fetched and processed the profile pages. As PlanetLab is a global network, each dataset contains GMT time for consistency reasons. The setup of our measurement along with the topology of the used PlanetLab nodes is presented in Figure 1. Any PlanetLab node can be out of order in any time, therefore we deployed the users to be monitored evenly between the nodes in order to minimise the damage of an unavailable node. As a consequence, we managed to monitor the vast majority of the selected users of the OSNs for more than three weeks continually. To reduce the size of the datasets, data of a user is only stored if the user is online.

\section{User Activity in Online Social Networks}

The members of online social networks may have different causes to use the services of the OSNs, accordingly each user has its own behaviour. In this section, we analyse our measurement results using statistical methods in order to classify the users of online social networks, furthermore we reveal the common characteristics of the members. First, we present some high-level statistics of our dataset. After that, we reveal the cluster structure of OSNs, finally we shows some interesting finding on how the users sign out from the sites.

\subsection{High-Level Statistics}

Our dataset contains user activity information on a minute basis, therefore highlevel statistics, including average uptime, downtime, and login frequency, can be computed. Uptime, or sojourn time, describes the time period when a user is 


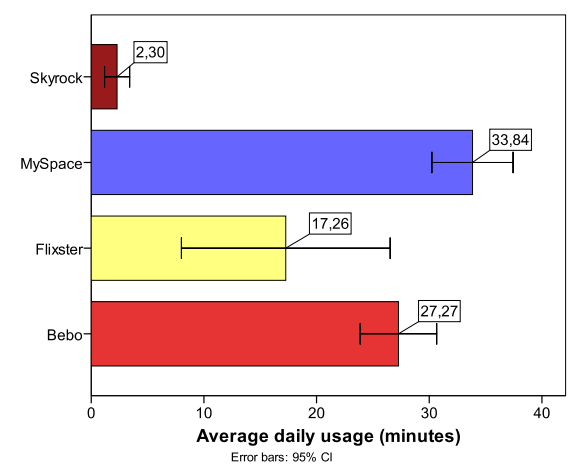

Fig. 2. Average daily usage time of the monitored users during our measurement

logged into an online social network, i.e. the time the user spent using the services of the OSN. Similarly, we use the term downtime for the time duration between two active sessions of a single user, while on login frequency we mean the number of logins of a single user during the measurement period.

The average daily usage time of the monitored users are shown in Figure 2. The users of MySpace have spent the most time on site while the members of SkyRock used their site the least. It is interesting, that data of Flixster has the highest variance. We note, that the measured average usage time depends on the way how the users leave the OSNs, Bebo has 30 minutes server time out, MySpace operates with 20-minute long sessions, while SkyRock handles a user offline after 7 minutes. However, these average sojourn times indicates that the monitored OSNs has different structure and services implying diverse user behaviours.

Detailed high-level statistics are presented in Table1, including uptime, downtime, and the frequency of the usage. The users of MySpace have spent 11.58 minutes on site after a login in average, much less than Flixster or Bebo users. However, the duration between two log-ins is the smallest at MySpace, in average the users are only 10 hours offline then they check their OSN. Accordingly, as the number of weekly sign-ins shows, the monitored population of MySpace was the most active while the activity of SkyRock users was moderate. The different usage patterns may represent the fascination of the social networks, in terms of popular services, number of friends, because a user spends more time on a more interesting site. The implication of these findings is that it is worthy to use similar services like MySpace has on a new OSN to have more active users, as active users produce revenue for the operator of the OSN.

\subsection{Identifying User Groups in Online Social Networks}

In the followings, we focus on the user population of MySpace and Bebo as we characterise the different groups of the OSNs, because we were able to monitor more users in these systems due to the availability of PlanetLab nodes during 
Table 1. High-level statistics of our online social network datasets

\begin{tabular}{c|c|c|c|c} 
& Bebo & Flixster & MySpace & SkyRock \\
\hline Number of monitored users & 954 & 451 & 942 & 422 \\
Average daily usage time (minutes) & 27.269 & 17.264 & 33.840 & 2.2963 \\
Standard deviation of daily usage & 53.437 & 100.094 & 56.3072 & 11.654 \\
Average uptime (minutes) & 28.15 & 18.20 & 11.58 & 9.17 \\
Standard deviation of uptime & 27.389 & 44.058 & 15.441 & 13.051 \\
Average downtime (days) & 1.011 & 1.044 & 0.334 & 3.985 \\
Standard deviation of downtime & 2.888 & 3.308 & 1.458 & 6.441 \\
Average weakly number of logins & 6.385 & 6.2253 & 20.043 & 1.341 \\
Standard deviation of weakly logins & 17.725 & 28.746 & 33.550 & 5.296
\end{tabular}

Table 2. Properties of centroids of the different clusters of MySpace

\begin{tabular}{c|c|c|c|c|c|c|c|c} 
& \multicolumn{9}{|c|}{ Cluster } \\
& 1 & 2 & 3 & 4 & 5 & 6 & 7 & 8 \\
\hline Uptime (mean) & 8.9721 & 34.77775 & 26.4393 & 17.1775 & 10.6759 & 8.7699 & 9.8596 & 5.7890 \\
Uptime (stddev) & 11.121 & 33.642 & 21.248 & 12.003 & 14.864 & 8.988 & 11.172 & 6.388 \\
Uptime (median) & 4.556 & 25.794 & 21.016 & 16.922 & 10.056 & 8.041 & 5.681 & 3.258 \\
Uptime (sum) & 2861.29 & 1927.47 & 1495.63 & 356.46 & 22.89 & 39.79 & 1018.36 & 153.98 \\
Downtime (mean) & 76.967 & 662.95 & 602.470 & 1511.56 & 11405.0 & 5519.93 & 247.83 & 1200.88 \\
Downtime (std. dev.) & 230.93 & 1438.79 & 1023.66 & 2056.54 & 8405.65 & 6277.05 & 578.51 & 2067.38 \\
Downtime (median) & 2.922 & 231.056 & 225.727 & 789.009 & 11714.8 & 3512.61 & 21.474 & 339.447 \\
Logins & 327.64 & 73.24 & 57.79 & 20.18 & 2.14 & 4.73 & 108.16 & 28.33 \\
Friends & 101.62 & 450.18 & 32.28 & 14.83 & 11.36 & 10.88 & 25.63 & 17.69 \\
Number of users in cluster & 45 & 17 & 97 & 160 & 36 & 86 & 152 & 227
\end{tabular}

our measurement. However, the members of Flixster and SkyRock can be also classified into several groups based on their behaviour.

The users of MySpace can be classified into eight groups based on their behaviour, we used the TwoStep clustering algorithm of the SPSS statistical software in our analysis. Each monitored user has its personal behaviour descriptors, like uptime, downtime, number of logins, for the duration of our measurement. Based on the datasets we calculate the users' individual statistics, including mean, standard deviation, median, sum, afterwards we compute the average properties which describe the user behaviour in the clusters. The detailed statistics are shown in Table 2, these values describe the centroids of the groups. The most diverse values of the properties are shown with italic characters.

In order to understand better the typical properties of the clusters, we visualize the relation between the average downtime and the uptime of the users along with the membership information in Figure 3, Figure 3(a) shows all the users while the more active user groups, in terms of shorter downtimes, are presented in Figure 3(b) Cluster 5 consists of users who are visiting their OSN very rarely, while members of Cluster 6 use MySpace a bit more frequently. Based on the average uptime, Cluster 2 consists of users who are online both for long periods 


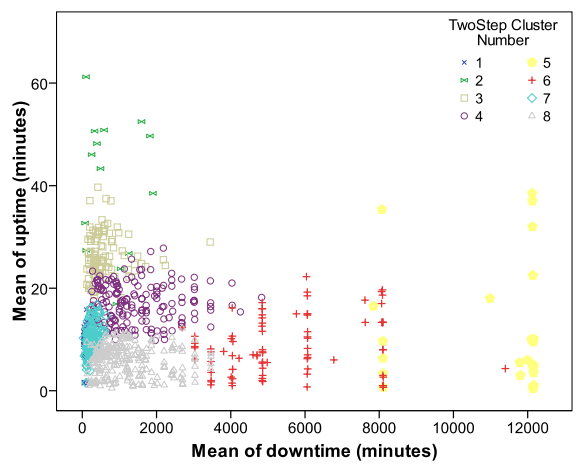

(a) All users

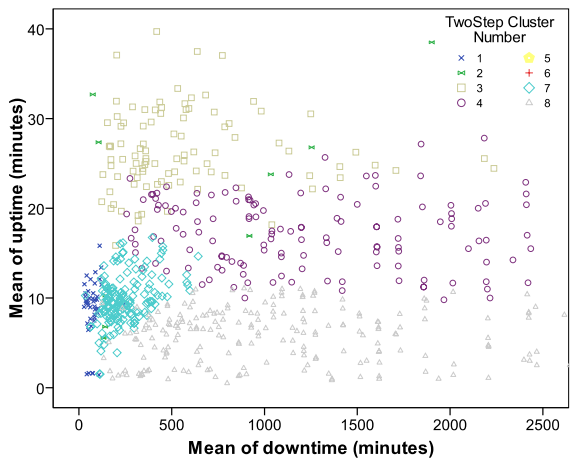

(b) Users with more frequent usage

Fig. 3. Members of different groups based on their average uptime and downtime periods [MySpace]
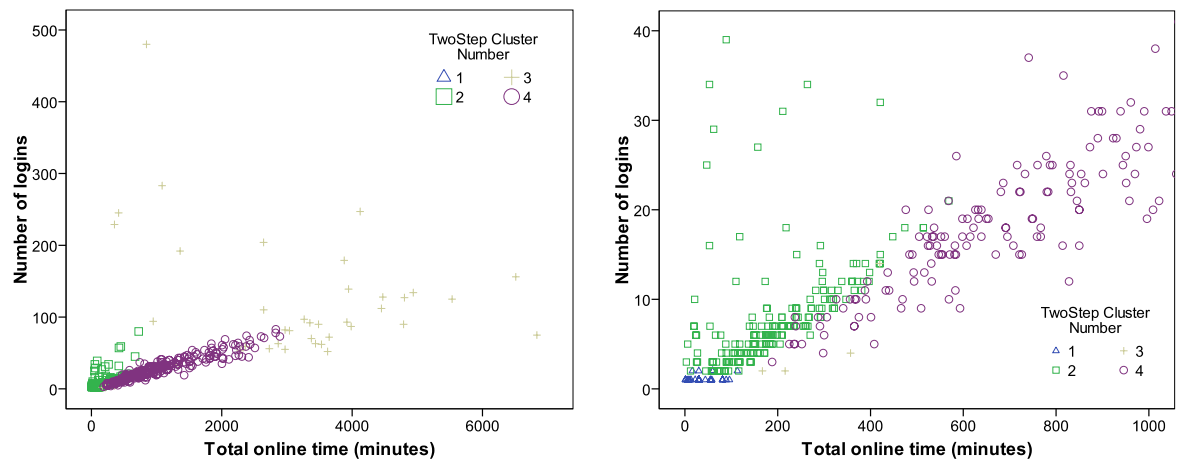

Fig. 4. Clustering users of Bebo

and frequently. In Figure 3(b), the characteristics of the remaining five groups are shown. Based on the time spent on site Cluster 3, 4, and 8 can be separated where the former is the group of most actives while the later's users are least "OSN dependent". The final two clusters (1 and 7) are separated based on their login frequencies, the users of Cluster 1 have the shortest downtime periods.

In Figure 5. five clusters out of eight are presented along with their best fitting cubic functions. Cluster 5 and 6 are distinguished based on the number of logins. These users check their pages around once a week, but if they are online they spend varying amount of time on the OSN. The deviation of uptime periods is an important factor dealing with user groups. Users with smaller deviation have used MySpace for shorter periods than users with larger deviation. These clusters have different user behaviour, therefore the members have to be treated distinctly to maximise the users' satisfaction. 


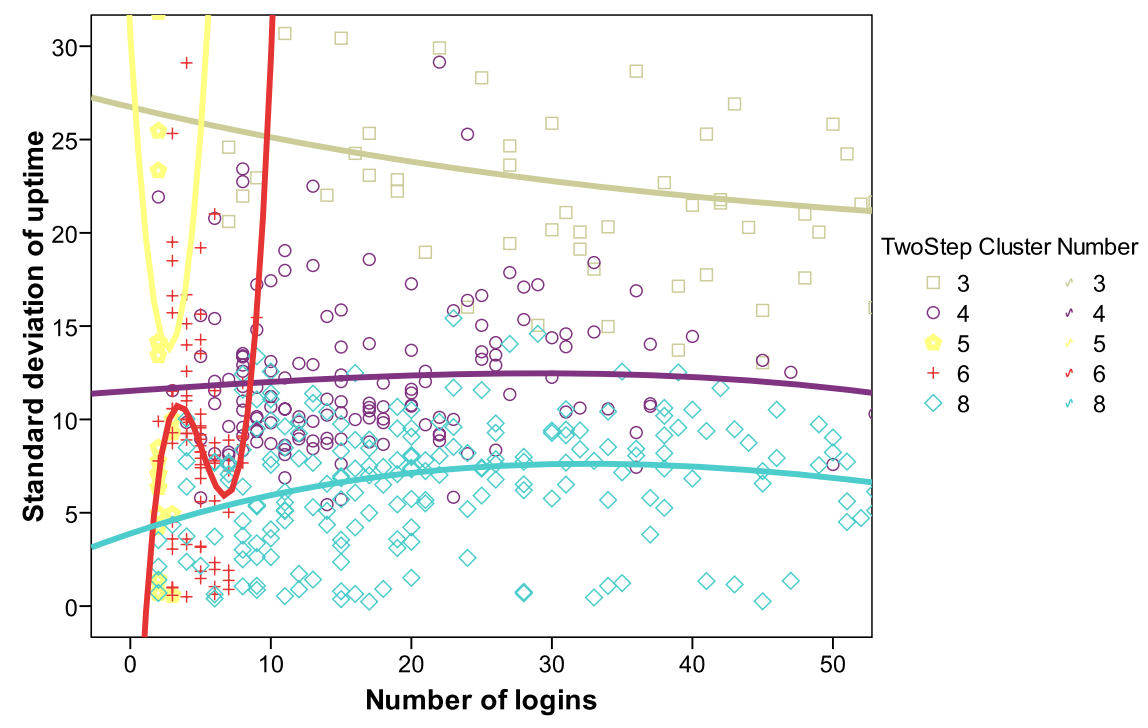

Fig. 5. Standard deviation of uptime periods based on the number of total logins

We use the same clustering algorithm to reveal user groups of Bebo. The sum of time spent online and the number of logins are presented in Figure 4 for Bebo users on two scales. Cluster 3 represents the heavy-users of Bebo, meaning they spend much more time online and/or they sign in to Bebo more frequently. These members are important for both the OSN operating company and for rival OSNs as well. On the one hand, the operator of the OSN have to pay special attention for the heavy users as these users love the services of the OSN, therefore they may persuade their friends to use that OSN. On the other hand, a rival OSN is interested in tempting these users to join their OSN, e.g. by sending special offers to the profile pages of the heavy users. These opposite interests have the same foundation as each OSN wants to earn more money by having more active users online. The members of Cluster 4 spend more time online than the users of Cluster 2, who rather check back more frequent. The last group is formed by users with little interest in Bebo services.

\subsection{Sign Out Methods}

The focus of our measurement method is the characterisation of user groups at different online social networks based on user activity. However, we are able to predict the way how the users left the OSN site purely based on our observations. As mentioned earlier, we have determined the length of the OSNs' time out session, i.e. the time after the site considers a user as being disconnected from the OSN. A user can left the OSN two different ways, sign out properly by 


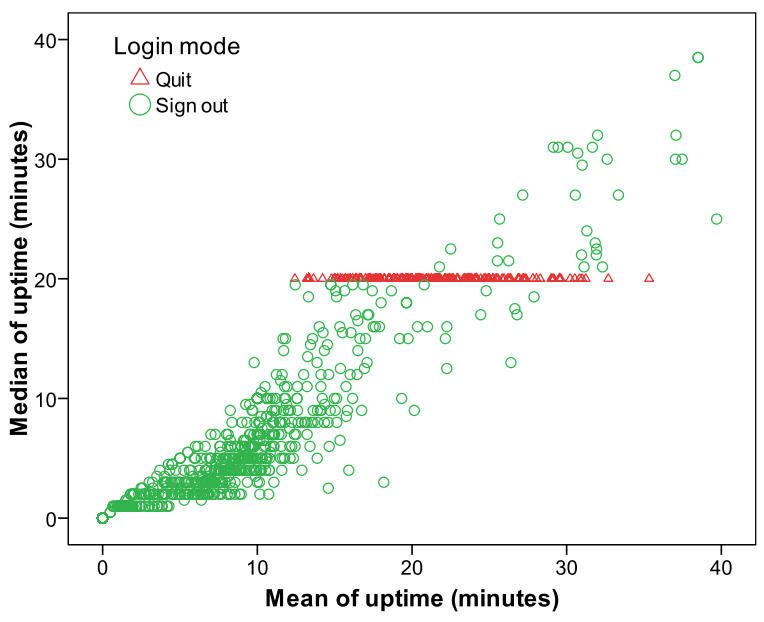

Fig. 6. Sign out methods

clicking the appropriate button or simply closing its Internet browser. In the later case, the session remains active until the time out, therefore it can cause security concerns.

MySpace notices immediately if a user signs out, however it has a 20-minute long session period. The implication of this property is strait forward, if the median of a user's uptimes is 20 minutes, than it is likely, that the user usually just closes its browser, instead of signing out properly. In this case, a short, less than a minute long online period is monitored as a 20-minute long uptime usage, because the user is treated as offline only after the server session times out. The median of the users' uptime as a function of the average uptime period is shown in Figure 6. We use median instead of mode because the uptime can have many values, therefore a value can be the mode of the sample even with only few occurrences. In this case, the median predicts better the users' behaviour. The users who close their browsers are illustrated, based on this method we argue that more than 25 percent of the users do not use the sign out button. We get almost the same ratio if we classify the users based on their modes. As a verification we look the most frequent uptimes in the dataset, the 20-minute activity is the second most frequent period (13.6\%), just after the 1-minute long usage $(20.7 \%)$.

\section{Conclusions}

In this paper, we have presented a detailed and in-depth analysis of user activity on some popular online social networks based on real world measurements. The characteristics of the activity of users on Bebo, Flixster, MySpace, and SkyRock have been analysed. We have identified several groups of OSN users, the characteristics of the clusters have been reviewed. Moreover, we observed that, similar 
to peer-to-peer systems, there exists a group of users in the OSNs, the heavy users, who use the sites really often for long periods. Our findings about different user groups with different user behaviours can be useful for OSN operators, who are willing to enhance their profit by keeping their heavy users, and also for developers of new OSNs, who can design their services incorporating solutions of popular OSNs to have lot of active users. Despite we have used only publicly accessible information in our measurement, we were able the extract personal online status information, which can be sensitive in some cases. We hope, that our work can initiate a discussion about privacy issues in OSNs, including the accessibility of online status information. Regarding future work, we plan to substantiate our measurement on more users and systems.

\section{Acknowledgement}

This paper has been partially supported by HSNLab, Budapest University of Technology and Economics, http://www.hsnlab.hu

\section{References}

1. PlanetLab, http://www.planet-lab.org/

2. Mislove, A., Marcon, M., Gummadi, K., Druschel, P., Bhattacharjee, B.: Measurement and Analysis of Online Social Networks. In: Proceedings of Internet Measurements Conference (2007)

3. Mislove, A., Koppula, H., Gummadi, K., Druschel, P., Bhattacharjee, B.: Growth of the Flickr Social Network. In: Proceedings of the First Workshop on Online Social Networks (2008)

4. Nazir, A., Raza, S., Chuah, C.: Unveiling Facebook: A Measurement Study of Social Network Based Applications. In: Proceedings of Internet Measurements Conference (2008)

5. Gjoka, M., Sirivianos, M., Markopoulou, A., Yang, X.: Poking Facebook: Characterization of OSN Applications In. In: WOSN 2008: Proceedings of the First Workshop on Online Social Networks (2008)

6. Cha, M., Kwak, H., Rodriguez, P., Ahn, Y., Moon, S.: I Tube, You Tube, Everybody Tubes: Analyzing the World's Largest User Generated Content Video System. In: Proceedings of Internet Measurements Conference (2007)

7. Chun, H., Kwak, H., Eom, Y., Ahn, Y., Moon, S., Jeong, H.: Comparison of Online Social Relations in Volume vs. Interaction: a Case Study of Cyworld. In: Proceedings of Internet Measurements Conference (2008)

8. Cha, M., Mislove, A., Adams, B., Gummadi, K.: Characterizing Social Cascades in Flickr. In: Proceedings of the First Workshop on Online Social Networks (2008)

9. MySpace, http://www.myspace.com

10. Bebo, http://www.bebo.com

11. SkyRock, http://www.skyrock.com

12. Flixster, http://www.flixster.com/

13. comScore Inc., http://www. comscore.com/

14. Economics of Networked Systems Group, BME, http://netecon_group.tmit.bme.hu/ 